\title{
Superposition method for the simulation of heat transfer
}

\author{
Y. Jiang ${ }^{1 *}$, Eric Li ${ }^{2}$, Q.X. Pei ${ }^{3}$, P. Liu ${ }^{3}$ and N. Sridhar ${ }^{3}$ \\ ${ }^{1}$ Hefei General Machinery Research Institute, No.888, West Changjiang Road, Hefei, Anhui, China \\ 230031 \\ ${ }^{2}$ School of Aerospace, Mechanical and Mechatronic Engineering, The University of Sydney, Sydney, NSW \\ 2006, Australia \\ ${ }^{3}$ Department of Engineering Mechanics, Institute of High Performance Computing, 1 Fusionopolis Way, \\ Connix, Singapore 138632
}

\begin{abstract}
Heat transfer plays a significantly important role in practical engineering. In this work, superposition method is applied to heat transfer simulation. In the superposition method, a representative element is selected. A pulse of heat is applied to the representative element to produce the data map by finite element method. The temperature history for the nodes of interest is recorded in the data map. To take the boundary effect into consideration, boundary elements are also selected to produce data maps. In the superposition method, time and space shifts are made in the data maps and the temperature increment is summed up. The temperature for the nodes of interest can be obtained. Good agreement is reached between the superposition method and the fullscale finite element method. The computational efficiency of superposition method is extremely higher than finite element simulation, especially for large-scale and long-time simulation.
\end{abstract}

Keywords: Superposition method; heat transfer; space shift; time shift; data map

*Corresponding author. E-mail address: 2731752093@qq.com 


\section{Introduction}

Heat transfer plays a vital role in engineering. Nearly all methods of power production involve heat transfer. Major segments of the chemical and metallurgical industries use components such as furnaces, heat exchangers, condensers and reactors. Aircraft and rocket owe their function partly to heat transfer. In microelectronics, heat transfer is often the limiting factor, which affects the duration and function of the chip. Since heat transfer has an overwhelming impact on engineering, it is of great significance to predict and control heat transfer. Prediction of heat transfer can be obtained by experimental investigation, theoretical calculation or numerical method. Experimental investigation is the most reliable tool. However, it is quite expensive and sometimes very difficult to realize. Analytical solutions only exist for some very simple cases. Therefore, numerical methods have been widely used in prediction of heat transfer. Among these numerical methods, finite element method (FEM), finite difference method (FDM) and finite volume method (FVM) are applied to heat transfer. Recently, a node-based smoothed finite element method (NS-FEM) has been developed in the meshfree framework and applied to heat transfer problems [1-4]. These numerical methods do work for heat transfer problems and show some advantages. However, heat transfer is a transient process, which needs matrix calculation and equation solution for each iteration. Using FEM or other above-mentioned numerical methods to run a longtime simulation, plenty of time and computation resource are needed. In order to reduce the computation cost, superposition method is applied to heat transfer in this work.

In the superposition method, the temperature can be obtained by the summation of the results of all the factors that can influence the temperature. The superposition method has been widely applied to practical engineering. Gorman and Sharma [5] presented the concept of the superposition method and its potential applications in 
obtaining accurate analytical solutions for rectangular thin plates with arbitrary combinations of classical boundary conditions. The superposition method was also extended to free flexural vibration of rectangular cantilever plates [6]. Koopmann [8] based on the principle of wave superposition computes the acoustic fields of arbitrarily shaped radiators. The results of several numerical experiments are presented that demonstrate the simplicity of the method. The superposition method was utilized to assess the thermal performance of heat sinks with one and two pairs of embedded heat pipes [7]. Transient heat conduction problems when initial temperature is non-uniform are solved with the help of superposition method [9-10]. In this work, the superposition method based on data maps is applied to simulate the temperature field. A representative element is first chosen and a unit pulse is applied to this element. The temperature history of the nodes in the model is recorded as a map. Using the data map, time and space shifts are made correctly to obtain the temperature increment of the node due to each pulse and the temperature increment induced by all the pulses in the loading profile is added up to get the temperature of the node. The boundary effect is also taken into consideration by choosing boundary elements to produce data maps. This superposition method based on data maps is applied to two examples to test the applicability of the method. Good agreements are reached between superposition method and full-scale finite element method. The efficiency is greatly increased by the superposition method especially for large-scale and long-time simulation.

This paper is organized as follows: a theoretical description of governing equation in three-dimensional heat transfer problem is presented in Section 2. Section 3 is the application of the superposition method to two examples. In Section 4, the conclusion is made. 


\section{Governing equation of heat transfer}

The governing equation of three-dimensional transient heat transfer can be described as follows:

$$
\frac{\partial^{2} T}{\partial x^{2}}+\frac{\partial^{2} T}{\partial y^{2}}+\frac{\partial^{2} T}{\partial z^{2}}+\frac{\dot{e}_{g e n}}{k}=\frac{1}{\alpha} \frac{\partial T}{\partial t}
$$

Here $T$ is the temperature field, which is a function of location and time, i.e. $T(x, y, z, t)$. $\alpha=\frac{k}{\rho c}$, where $k$ is the thermal conductivity of the medium. $c$ is the specific heat. $\rho$ is the material density. $\dot{e}_{g e n}$ is the heat generation rate of heat source.

There are three types of boundary conditions. The first type of boundary condition is that on certain surface the temperature field is known. It is expressed as:

$$
T(x, y, z, t)=\varphi_{s}(x, y, z, t)
$$

$\varphi_{S}(x, y, z, t)$ is the known temperature field on the surface.

The second type of boundary condition is that the heat flux on certain surface is known. It is expressed as:

$$
k \frac{\partial T}{\partial x} n_{x}+k \frac{\partial T}{\partial y} n_{y}+k \frac{\partial T}{\partial z} n_{z}=q_{s}(x, y, z, t)
$$

Here $n_{x}, n_{y}$ and $n_{z}$ are the components of outward unit norm along the surface in $x, y, z$ direction, respectively. $q_{S}(x, y, z, t)$ is the heat flux on the surface. The simplest case is that the heat flux is zero, which means that the surface is adiabatic.

The third type of boundary condition is that there exists heat exchange between the surface and the surrounding medium. The equation is expressed as:

$$
k \frac{\partial T}{\partial x} n_{x}+k \frac{\partial T}{\partial y} n_{y}+k \frac{\partial T}{\partial z} n_{z}=\beta\left(T_{\alpha}-T_{s}\right)
$$


Here $\beta$ is the heat exchange coefficient between the surface and the surrounding medium. $T_{\alpha}$ is the temperature of the surrounding medium and $T_{S}$ is the temperature of the surface.

Besides the boundary condition, in order to solve the governing equation, the initial condition has to be known. The initial condition is the temperature field $T(x, y, z, t)$ of the whole model at $t=0$.

$$
T(x, y, z, 0)=\varphi(x, y, z)
$$

Here $\varphi(x, y, z)$ is the temperature field of the whole model at $t=0$.

\section{Examples}

In order to show how the superposition method is applied to heat transfer and test whether superposition method is applicable to heat transfer, superposition method is applied to two examples. The first example is a cubic of aluminum with heat source inside. Heat exchange exists between the cubic surfaces and the air. The second example is a thermal printer.

\subsection{Application of the superposition method to an aluminum cubic}

\subsubsection{Finite element model}

In this case, a $1 \mathrm{~mm} * 1 \mathrm{~mm} * 1 \mathrm{~mm}$ aluminum cubic with a $5 * 5 * 15$ mesh is used, shown in Fig. 1. In the simulation, three heater elements E6, E7 and E8 are loaded. The loading profiles for these three heater elements are plotted in Figs. 2(b)、2(c)、 2(d). The boundary condition is set as follows: heat exchange between the boundary of the cubic and the air. The ambient temperature is considered to be constant as $24{ }^{\circ} \mathrm{C}$. The initial temperature of the whole cubic is $24{ }^{\circ} \mathrm{C}$. The heat exchange coefficient between the surface and the air is $6 \mathrm{~W} /\left(\mathrm{m}^{2 *} \mathrm{~K}\right)$. The material property of aluminum is as follows: the density is $2680 \mathrm{~kg} / \mathrm{m}^{3}$, the thermal conductivity is $167 \mathrm{~W} /(\mathrm{m} * \mathrm{~K})$ and the 
specific heat is $880 \mathrm{~J} /\left(\mathrm{kg}^{*} \mathrm{~K}\right)$. The heat transfer process is simulated by ABAQUS. Each sub-step is set as $0.01 \mathrm{~ms}$. Implicit solver is used. The temperature evolution of the nodes is obtained and plotted in Fig. 3.

\subsubsection{Superposition method}

The superposition method is also applied to obtain the temperature evolution of the nodes. In the superposition method, element E8 located in the middle of the cubic is selected as a representative element. A unit pulse lasting $0.01 \mathrm{~ms}$, shown in Fig. 2(a), is applied to the representative element with the same boundary condition and initial condition as full-scale simulation in ABAQUS. The temperature increment of the nodes versus time is recorded in a data map shown in Table 1 . In the table, the row labeled ' $\mathrm{N} 8$ ' is the temperature increment of node $\mathrm{N} 8$ at different time after the unit pulse is applied to the representative element E8. It is seen that at $\mathrm{t}=0.01 \mathrm{~ms}$ the temperature increment of node $\mathrm{N} 8$ is $0.57{ }^{\circ} \mathrm{C}$, which is one of the two biggest values in the table. The other biggest value is the temperature increment of node N9 at the same time. This is reasonable because nodes N8 and N9 are symmetrical with respect to element E8. Thus the unit pulse applied to element E8 will increase the temperature of nodes N8 and N9 by the same degree. As time goes by, the temperature of nodes N8 and N9 becomes smaller due to the heat dissipated to the surrounding elements, and the temperature increment of the surrounding nodes are increasing as time goes by.

Now let us describe how to apply the superposition method to obtain the temperature of the node due to loading profiles shown in Fig. 2 based on the data map produced above. For example, the temperature increment of node N7 at $\mathrm{t}=0.06 \mathrm{~ms}$ due to the three firing elements E7, E8 and E9 is to be calculated. Before $0.06 \mathrm{~ms}$, there are three pulses donated by P1, P2 and P3 applied to element E8, shown in Fig. 2(c). For pulse $\mathrm{P} 1$, which lasts from $0.02 \mathrm{~ms}$ to $0.03 \mathrm{~ms}, \mathrm{t}=0.06 \mathrm{~ms}$ is $0.03 \mathrm{~ms}$ away. Thus the 
temperature increment of node N7 due to pulse P1 is picked from N7 row and column $0.04 \mathrm{~ms}(0.01 \mathrm{~ms}+0.03 \mathrm{~ms})$, i.e. $0.12^{\circ} \mathrm{C}$. $0.06 \mathrm{~ms}$ is $0.01 \mathrm{~ms}$ away from pulse $\mathrm{P} 2$. Therefore a $0.01 \mathrm{~ms}$ time shift is made in the data map in row $\mathrm{N} 7$, and $0.08^{\circ} \mathrm{C}$ is picked from the table. Similarly, for pulse P3 the temperature increment is $0.05{ }^{\circ} \mathrm{C}$. The total temperature increment of node N7 due to these three pulses from element E8 is the sum of these three terms $\left(0.12+0.08+0.05=0.25{ }^{\circ} \mathrm{C}\right)$.

To calculate the temperature increment induced by the heat generated by element E7, besides a time shift, a space shift is also needed. The relative location of node N7 with respect to element $\mathrm{E} 7$ is the same as that of node N8 to element E8. In superposition method, it is assumed that the temperature increment of node N7 caused by element E7 is the same as that of node N8 caused by element E8 under the same loading profile. Thus in the table, the temperature increment information in row ' $\mathrm{N} 8$ ' is taken as the temperature increment of node N7 caused by the pulses applied to element E7. After a time shift is made, the temperature increment induced by pulse $\mathrm{P} 1$ is $0.42{ }^{\circ} \mathrm{C}$. For pulse $\mathrm{P} 2$ the temperature increment is $0.46{ }^{\circ} \mathrm{C}$. For pulse $\mathrm{P} 3$, the temperature increment is $0.57{ }^{\circ} \mathrm{C}$. Thus the total temperature increment of node $\mathrm{N} 7$ at $\mathrm{t}=0.06 \mathrm{~ms}$ induced by the three pulses applied to element E7 is $0.42+0.46+0.57=1.45^{\circ} \mathrm{C}$.

Similarly, the total temperature increment of node N7 induced by the two pulses applied to element $\mathrm{E} 9$ is taken from row labeled ' $\mathrm{N} 6$ ', i.e. $0.02+0=0.02^{\circ} \mathrm{C}$.

Above all, the total temperature increment of node $\mathrm{N} 7$ at $\mathrm{t}=0.06 \mathrm{~ms}$ caused by the heat generated from elements E6, E7 and E8 is $0.25+1.45+0.02=1.72^{\circ} \mathrm{C}$, and the temperature of node $\mathrm{N} 7$ at $\mathrm{t}=0.06 \mathrm{~ms}$ is $24+1.72=25.72{ }^{\circ} \mathrm{C}$. In the same way, the temperature of node N7 and other nodes at any time can be calculated. The temperature of nodes N5, N6, N7, N8, N9 and N10 provided by ABAQUS and the superposition 
method is plotted and compared in Fig. 3. In the figure, '-FEM' stands for the result by ABAQUS, and '-sup' stands for result by the superposition method. From the comparison, it is seen that the result by ABAQUS and the superposition method is nearly the same. This proves that the superposition method is applicable to heat transfer in three-dimension.

\subsubsection{Boundary effect}

In the superposition method, the space shift is based on the assumption that the heat transfer condition of other elements is the same as the representative element. This assumption is valid only when the domain is infinite and the domain is uniformly meshed. But in reality, the domain is not infinite and has its boundary. The assumption is not available. In order to take the boundary effect into consideration, the boundary effect has to be investigated first. Here the boundary elements E1, E2 and E3 are taken to produce data maps. A unit pulse same as Fig. 2(a) is applied to the three elements respectively. The temperature increment of the nodes as time goes by is stored in the data maps separately, shown in Tables 2-4.

In order to make the boundary effect more obvious, the row of elements labeled "E1" to "E15" in Fig. 1 are all firing according to different loading profiles. In order to limit the length of the paper, the loading profiles are not plotted here. The boundary condition is the same as above. The temperature of node N1 by ABAQUS is plotted in Fig. 4, denoted by 'FEM'. Superposition method is also applied to obtain the temperature of node N1 at different time. At this time, besides the data map produced by element E8, the data maps produced by elements E1, E2 and E3 are also used. Firstly, the data map produced by the middle element E8 and the boundary element E1 are used. By this scheme, which is denoted by 'bound-1' in Fig. 4, the temperature increment of nodes induced by firing elements is obtained as follows: the temperature increment 
induced by element E1, is obtained from the data map produced by element E1. Elements E15 and E1 are symmetric. Therefore, the temperature increment induced by element E15 can be obtained by data map generated from element E1 and make corresponding symmetrical operation. When other elements are firing, the temperature increment of nodes is obtained from the data map produced by the middle element E8 with a space shift and a time shift.

In Fig. 4, 'bound-2' stands for data maps produced by boundary elements E1, E2 and E8. Similar to the scheme 'bound-1', the temperature increment induced by E1 and E2 is obtained from the data maps of E1 and E2 respectively. Symmetrical operation is made in the data maps of E1 and E2 for the temperature increment induced by elements E14 and E15. Similarly, 'bound-3' stands for data maps produced by elements E1, E2 and E3 are used as well as the middle element E8. 'bound-0' stands for the scheme using only the data map produced by E8. The results of these schemes and FEM are plotted and compared in Fig. 4. From the figure, it is seen that as more boundary element maps are employed, the results of superposition method are closer to the FEM results. This signifies the importance of boundary effect. When three boundary element data maps are employed in the scheme denoted by 'bound-3', the results are very close to the FEM results. It is also seen that as time goes by, the difference between the superposition method results and the FEM results is larger when 'bound-0', 'bound-1' and 'bound-2' are used. This can be explained by that as time goes by, more heat is dissipated to the boundary elements and boundary effect becomes more obvious.

From the results shown above, it is seen that boundary effect is obvious. Thus it is important to consider the boundary effect in the superposition method. To consider the boundary effect, data maps produced by boundary elements are needed. With the data maps from the boundary elements, superposition method is applicable to heat transfer 
simulation. It is also seen that the more data maps from the boundary elements, the closer the results of the superposition method are to the results of FEM.

\subsection{Application of the superposition method to the thermal transfer printer}

In the thermal transfer printer, heat transfer and temperature control are very important. Many works on one-dimensional or two-dimensional theoretical or numerical models have been reported. A simplified quasi 1-D mathematical model [11] to produce the temperature in the thermal printer system is developed. A sequence of mathematical models [12] describing the electro-thermal ribbon printing process are developed. The models describe the electrical processes, heat generation and heat transfer within the ribbon and the transfer of ink from the ribbon to paper. Simulation [13] involving computational fluid dynamics (CFD) and heat transfer phenomena is carried out with finite element method in order to optimize the device's elements shape. A twodimensional numerical model [14] which represents a micro-heater cross-section, gives the spatiotemporal evolution of the temperature field which highlights clearly the thermal loading phenomenon in the micro-heaters. Recently, a three-dimensional finite element model for thermal transfer printer is proposed [15], shown in Fig. 5. In this model, heat accumulation of multiple pages printing, different printing speed effect and paper movement simulation are included. The temperature of the 8 locations shown in Fig. 6, which is important and helpful in the optimization of the printer design, is measured by experiment. The results of the proposed model agree with the experimental results, shown in Fig. 7. It is observed that the proposed model is feasible and useful in the design of the thermal transfer printer. But this model is very large, consisting of 85,069 DC3D8 elements and 12,782 DC3D6 elements. The sub-step is $50 \mu \mathrm{s}$. It takes 12 parallel cpus around 7 hours to finish the simulation of one page printing and its following cooling down. If the simulation of 100 pages printing and cooling down 
process is to be finished, it will take 12 parallel cpus approximately one month. It is very time consuming and computationally expensive to run the three-dimensional heat transfer simulation and will delay the design of the printer. Thus it is important and significant to develop a faster method to obtain the temperature of the locations we are interested in. In view of the application of superposition method to the heat transfer simulation and the uniformity of the mesh in the model, superposition method is intended to be used in the temperature prediction of the 8 locations shown in Fig. 6. From Section 3.1.3 it is seen that the boundary effect is obvious. To include the boundary effect, data maps from boundary elements are needed.

There are totally 1248 heater elements in the printer. In the FEM model, each heater element is meshed with one element. Due to symmetry, only half of the printer is simulated with 624 heater elements. Here one element in the middle and four boundary elements are selected to produce data maps, shown in Fig. 8. From the figure, it is seen that No. 1, No. 104, No. 312, No. 520 and No. 624 elements in the row of firing elements are selected. No. 1 element is to represent the behavior of elements ranging from No. 1 to No. 52. Because the size of the firing element is very small $(85 \mu \mathrm{m}$ in width), No. 1 and 2 elements are very close to each other. The temperature increment of the nodes far away from elements No 1 and No 2 is nearly the same, no matter whether No. 1 or No. 2 element is firing. If No. 1 and 2 elements are both selected to produce data maps, it will cause a waste of time and computational resource. Thus No. 104 element is selected as the second element to represent elements from No. 53 to 156 . No. 312 element in the middle of the row represents the majority of the firing elements from No. 157 to 468 . Due to the difference of the boundary condition on the left side and the right side, another two elements (No 520 and 624) are also selected to produce data maps. No. 520 represents elements ranging from No. 469 to 573 and No. 624 represents 
elements from No. 574 to 624 . This representative elements selection scheme shows to be effective through our calculation.

After the five representative elements are selected, a unit pulse is applied to the five representative elements separately with other firing elements powered off to produce the data maps. The boundary condition is the same as that of the full-scale finite element simulation. Then the simulation runs as long as 200 pages printing, which can be used to predict 200 pages printing. After the simulation is finished, the temperature increment of nodes, which is needed in the superposition method to calculate the eight locations, is recorded in the data maps. Due to the large size of the data maps, we do not list them here. Here we have to point out that in order to reduce the numerical round off error, the magnitude of the unit pulse used to produce the data map is 10,000 times the actual magnitude of the pulse. Thus the temperature increment of nodes from the simulation has to be divided by 10,000 to produce data map. In the data map, if the temperature increment at each sub-step is to be recorded, the data volume is very large. In order to reduce the volume of the data map, the results for the first 10,000 sub-steps after the unit pulse are recorded because during this period of time, the temperature variation is large. After the initial 10,000 sub-steps, the temperature increment of nodes is recorded at every 1000 sub-steps. For the sub-step in between two neighboring integer time of 1000 sub-steps, the temperature increment can be obtained by linear interpolation of the temperature increment of two neighboring integer time of 1000 sub-steps. After the five data maps are produced, superposition method is used to get the temperature evolution of eight locations in the printer. The implementation of superposition method is as follows:

Loop over pages you want to print $n=1,2, \ldots$;

Loop over sub-steps $\mathrm{s}=1,2, \ldots$; 
Loop over the heater element $\mathrm{e}=1,2, \ldots, 624$;

If element e is firing for sub-step s; continue; else go to next heater element Find the appropriate data map for element e;

Make a space shift;

Loop over the locations $1=1,2, \ldots, 8$

Loop over the sub-steps after sub-step s,...;

Make a time shift to sub-step sn;

If sn is exactly an integer time of 1000; Obtain the temperature increment; else obtain the temperature increment of its two neighboring integer time of 1000 sub-steps and calculate the temperature increment of sn by linear interpolation;

Add up the temperature increment;

End of loop;

End of loop;

End of loop;

End of loop;

End of loop;

Loop over the locations $1=1,2, \ldots, 8$;

Loop over sub-steps $\mathrm{s}=1,2, \ldots$;

Add the temperature increment of each sub-step to the ambient temperature;

End of loop;

End of loop;

The procedure listed above can be realized by a Fortran code. By running this code the temperature of the eight locations can be obtained. The results of different cases by superposition method are plotted and compared with the experimental results, shown in 
Figs. 9-10. From the figures, it can be seen that the superposition method results agree with experimental results. This demonstrates the applicability of the superposition method to heat transfer problems. The most important thing is that the efficiency of the simulation is greatly improved. For example, it takes 12 cpus 30 days to run 100 pages simulation by ABAQUS. But by the superposition method, it takes $1 \mathrm{cpu}$ around 4 hours to finish the calculation. The efficiency is improved by more than 2000 times.

\section{Conclusion}

In this work, the superposition method is applied to heat transfer. In the superposition method, representative elements are selected to produce data maps. The temperature increment of nodes is obtained by summation of all the increments induced by all the heating elements. The results of the superposition method are compared with the full-scale finite element method or experimental results. Some conclusions are drawn as follows:

1. Superposition method is applicable to heat transfer problems. In the superposition method, representative elements are selected to produce data maps. Time and space shifts are made to obtain the temperature evolution with time.

2. Boundary effect is obvious so that boundary elements are selected to produce data maps. The more boundary element maps employed in the superposition method, the closer the results of superposition method are to the full-scale simulation results.

3. The efficiency of superposition method is significantly higher than full-scale finite element method especially for large-scale and long-time simulation. 


\section{REFERENCE}

[1] S.C. Wu, G.R. Liu, H.O. Zhang, X. Xu, Z.R. Li. A node-based smoothed point interpolation method (NS-PIM) for three-dimensional heat transfer problems. International Journal of Thermal Sciences 2009; 48:1367-1376.

[2] S.C. Wu, G.R. Liu, H.O. Zhang, G.Y. Zhang. A node-based smoothed point interpolation method (NS-PIM) for thermoelastic problems with solution bounds. International Journal of Heat and Mass Transfer 2009; 52:1464-1471.

[3] E. Li, G.R. Liu, T. Vincent, Z.C. He. An efficient algorithm for phase change problem in tumor treatment using $\alpha$ FEM. International Journal of Thermal Sciences 2010; 49 (10):1954-1967.

[4] E. Li, G.R. Liu, T. Vincent, Z.C. He. Modeling and simulation of bioheat transfer in the human eye using the $3 \mathrm{D}$ alpha finite element method $(\alpha \mathrm{FEM})$. International Journal for Numerical Methods in Biomedical Engineering 2010; 26: 955-976.

[5] D.J. Gorman, R.K. Sharma. A comprehensive approach to the free vibration analysis of rectangular plates by use of the method of superposition. Journal of Sound and Vibration 1976; 47(1):126-128.

[6] D.J. Gorman. Free vibration analysis of cantilever plates by the method of superposition. Journal of Sound and Vibration 1976; 49(4):453-467.

[7] J.C. Wang. Superposition method to investigate the thermal performance of heat sink with embedded heat pipes. International Communications in Heat and Mass Transfer 2009; 36:686-692.

[8] G.H. Koopmann, L.M. Song, J.B. Fahnline. A method for computing acoustic fields based on the principle of wave superposition. Journal of the Acoustical Society of America 1989; 86(6):2433-2438. 
[9] A.I. Pekhovitch, W.M. Dzjidkih. Calculations of thermal states of solid bodies (in Russian). Leningrad, Energija. 1976.

[10] M.M. Smirnoff. Exercises of math physics equation (in Russian). Nauka, Moscow. 1975.

[11] R. Uyhan, J.A. King-Hele. Modelling of thermal printers. Applied Mathematical Modelling 2008; 32:405-416.

[12] David N.M. Ibrahim, Richard J. Cameron. A model of the electrothermal ribbon printing process. Applied Mathematical Modelling 2006; 30:1561-1570.

[13] M. Iliescu, E. Nutu, B. Comanescu. Applied finite element method simulation in 3D printing. International Journal of Mathematics and Computers in Simulation 2008; 2(4):305-312.

[14] F. Lemeunier, N. Allnic, S. Belhabib, P. Mousseau and C. Plot. Experimental investigation and numerical modeling of the thermal behavior of a micro-heater. Microsystem Technologies 2016; 22(1):181-191.

[15] Y. Jiang, Eric Li, Q.X. Pei, P. Liu. Simulation of three-dimensional heat transfer in the thermal transfer printer (submitted). 


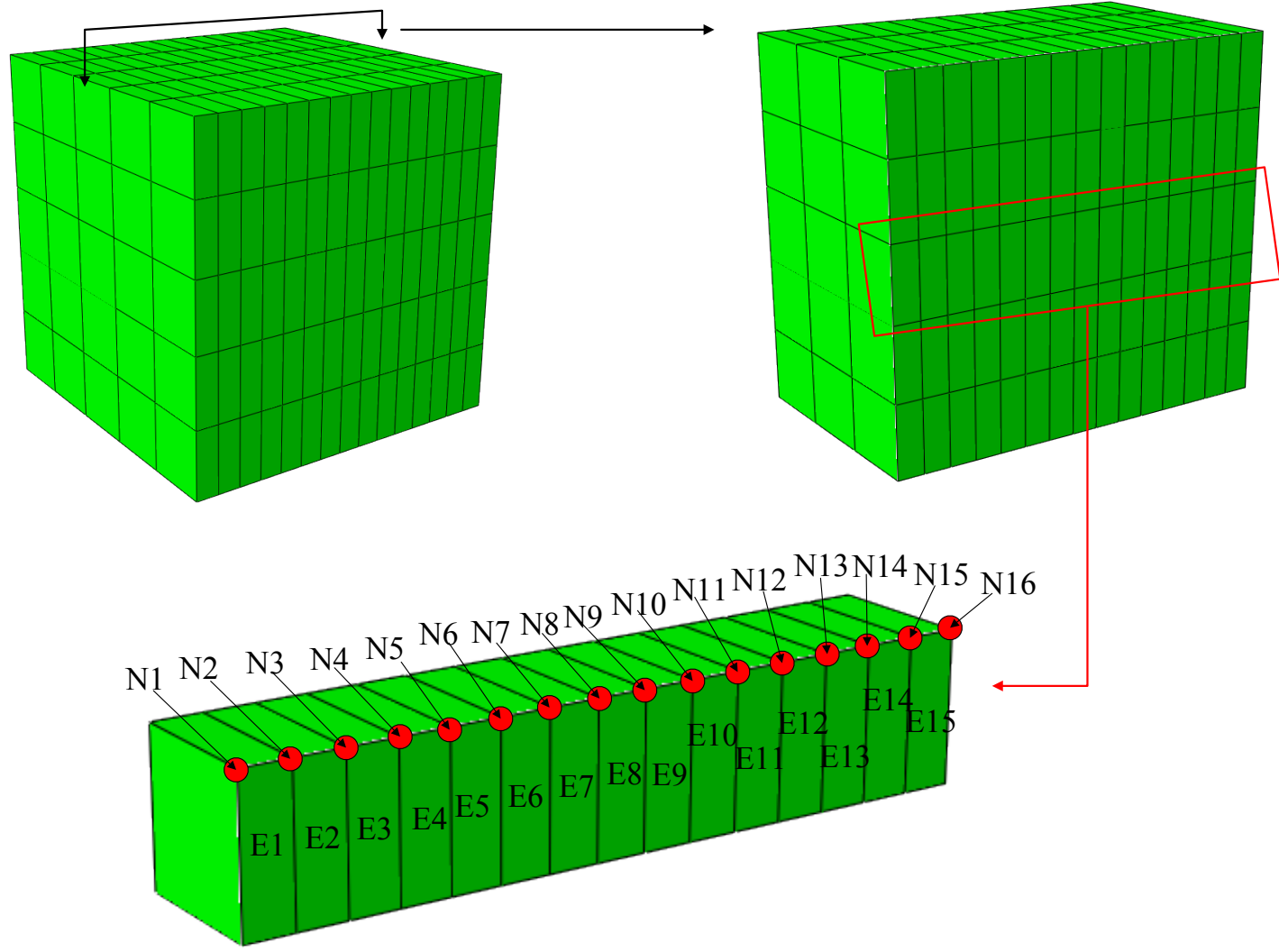

Fig. 1.The mesh of the cubic with node and element labels
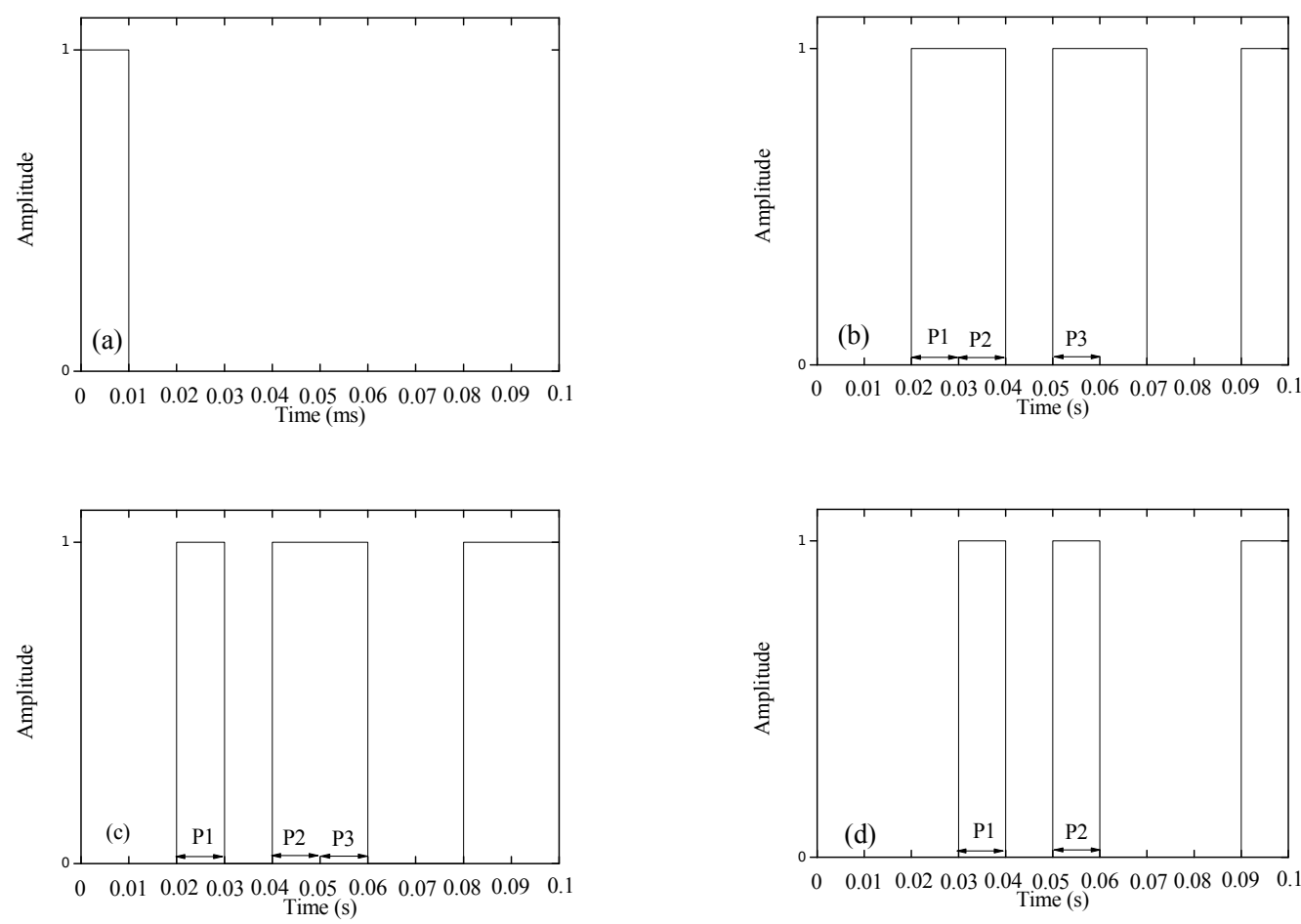

Fig. 2. Loading profiles: (a) a unit pulse applied to the representative element (b) loading profile applied to element E7 (c) loading profile applied to element E8 (d) loading profile applied to element E9 


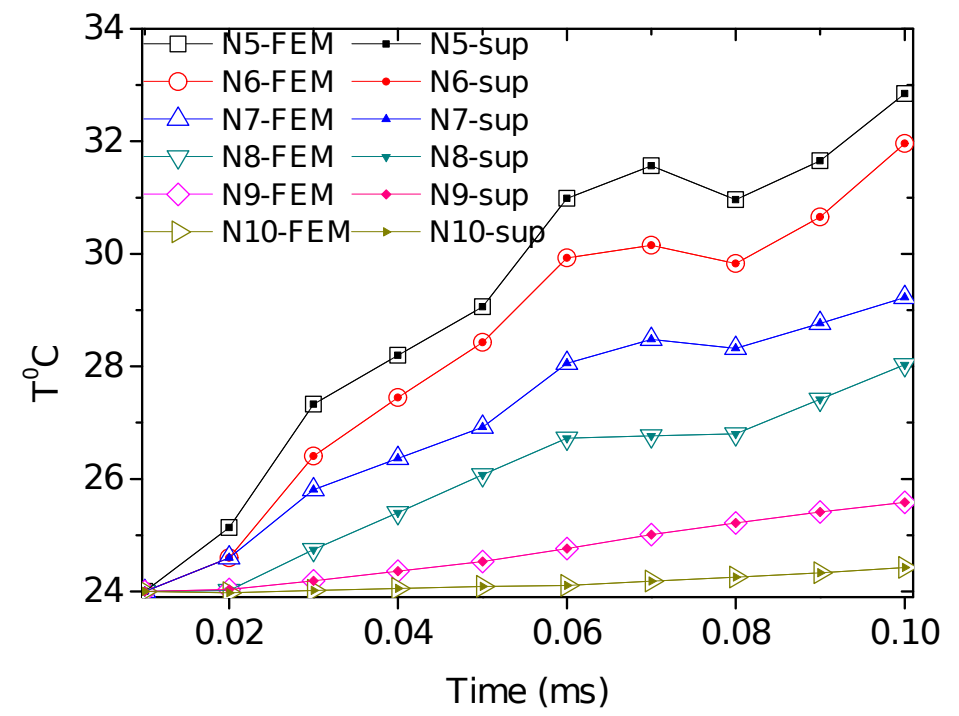

Fig. 3.The temperature evolution of nodes calculated by FEM and superposition method

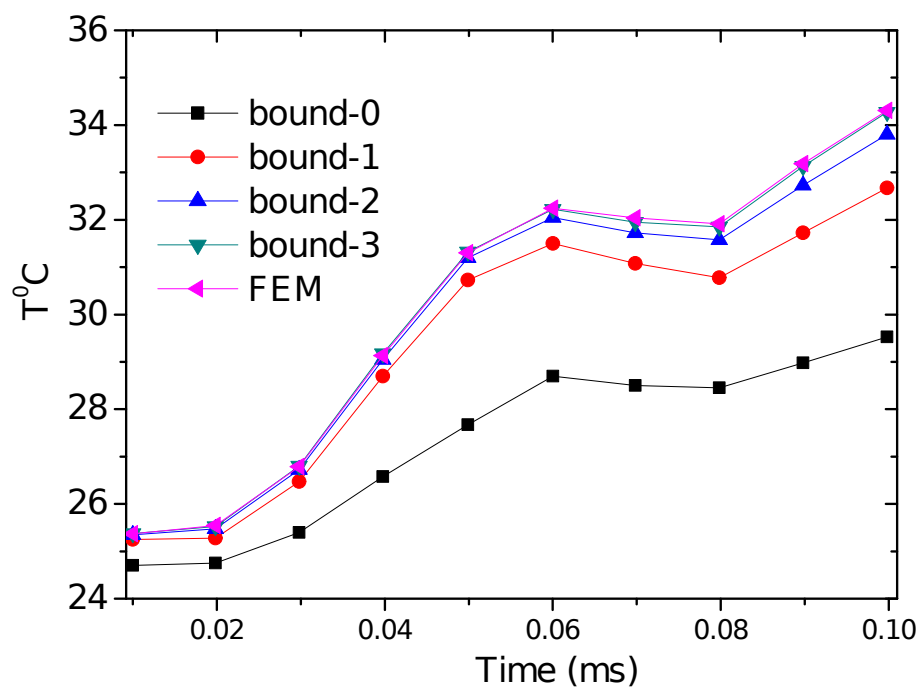

Fig. 4. The temperature evolution of node N1 by different schemes of superposition method and FEM 


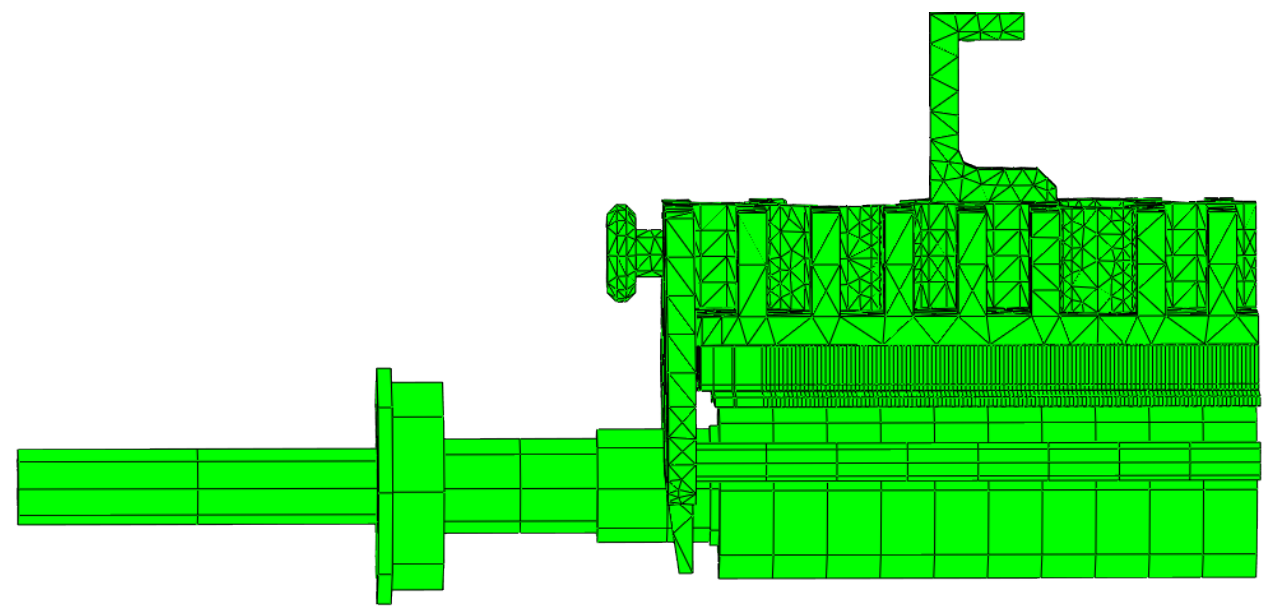

Fig. 5. The mesh of the thermal transfer printer
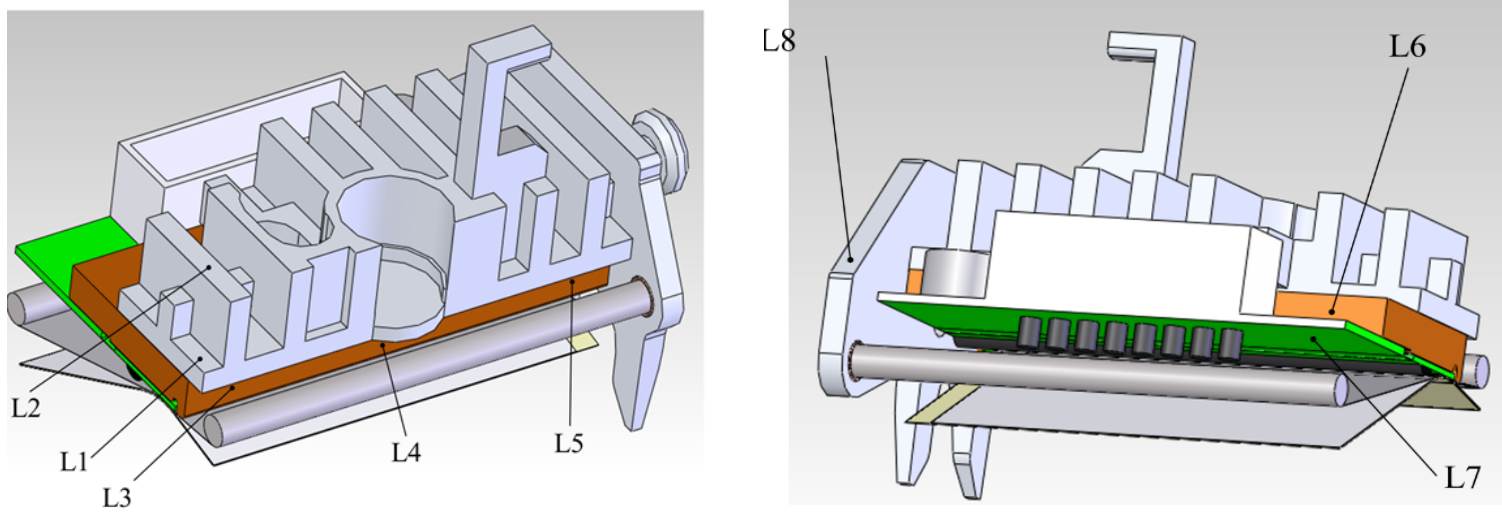

Fig. 6. The locations of eight points measured experimentally
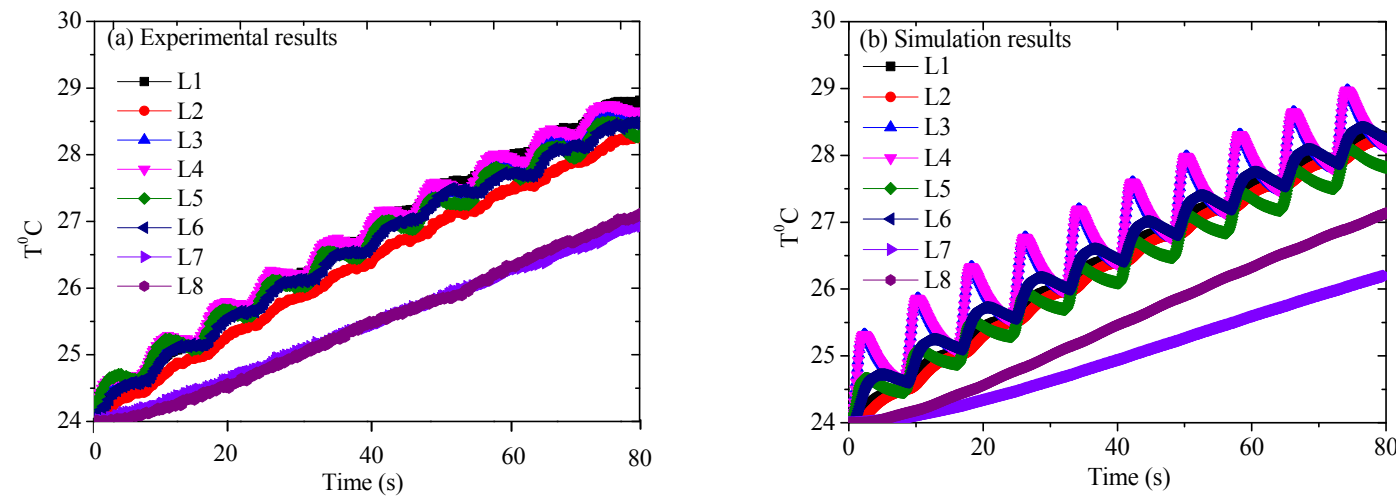

Fig. 7. Comparison between the experimental results and simulation results for ten pages printing at 5 ips with ambient temperature $24{ }^{\circ} \mathrm{C}$ 


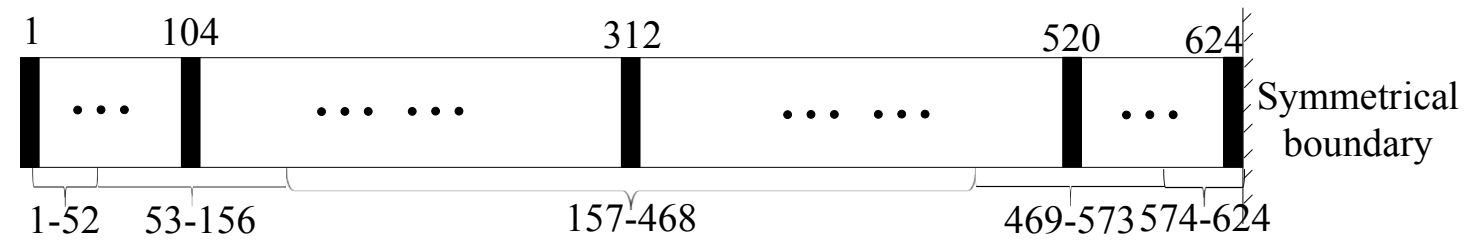

Fig. 8. The five representative elements selected to produce data maps and their covering ranges
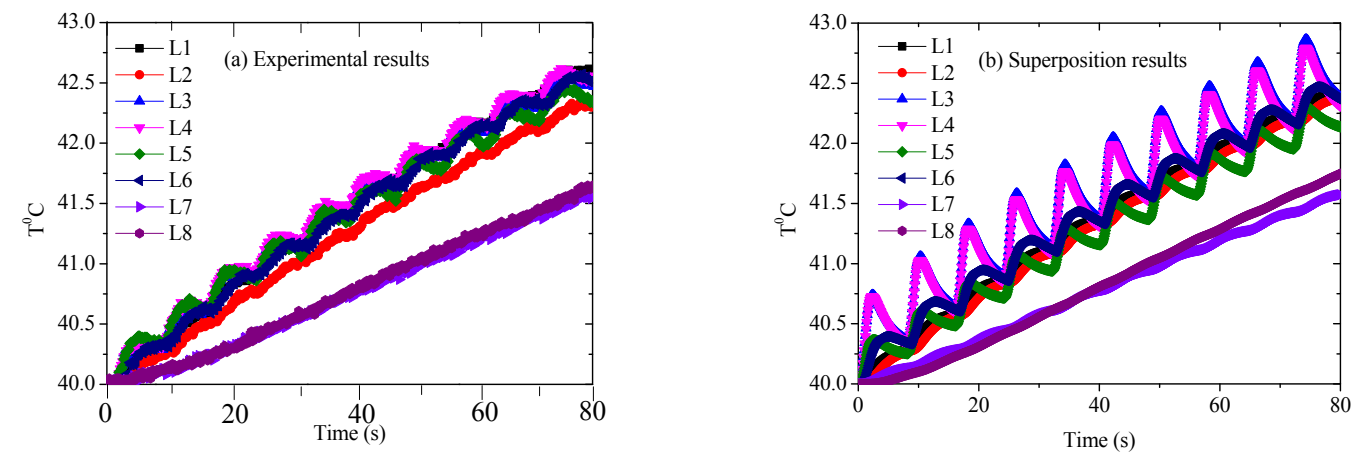

Fig. 9. Comparison between the experimental results and superposition results for ten pages printing at 5 ips with ambient temperature $40{ }^{\circ} \mathrm{C}$
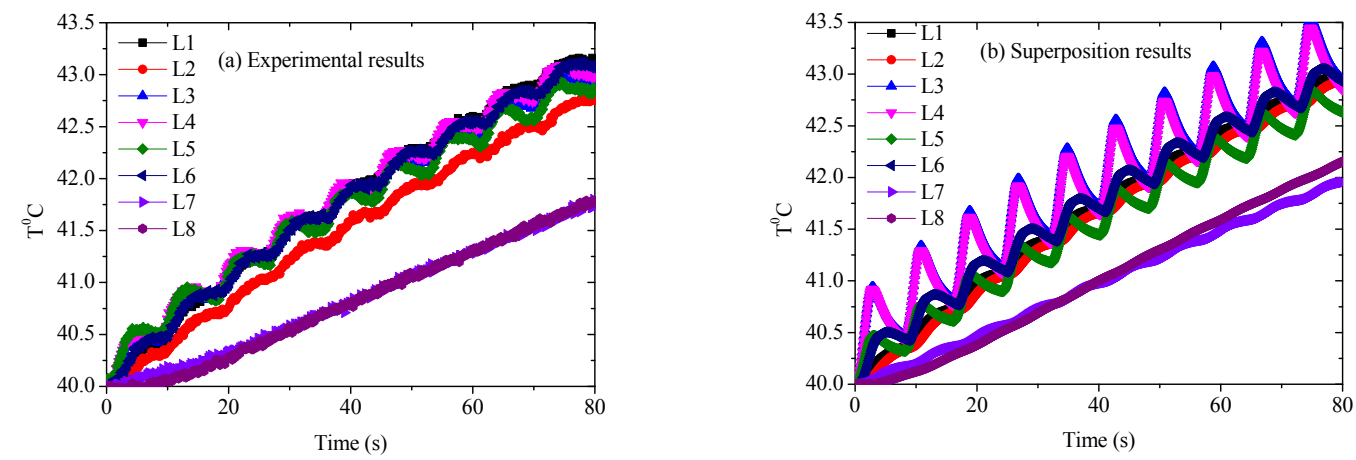

Fig. 10. Comparison between the experimental results and superposition results for ten pages printing at 3 ips with ambient temperature $40{ }^{\circ} \mathrm{C}$ 
Table 1. The data map produced by a single pulse applied to element E8

\begin{tabular}{|c|c|c|c|c|c|c|c|c|c|c|}
\hline $\mathrm{t}(\mathrm{ms})$ & 0.01 & 0.02 & 0.03 & 0.04 & 0.05 & 0.06 & 0.07 & 0.08 & 0.09 & 0.1 \\
\hline $\mathrm{N} 1$ & 0 & 0 & 0 & 0 & 0 & 0 & 0 & 0 & 0 & 0 \\
\hline $\mathrm{N} 2$ & 0 & 0 & 0 & 0 & 0 & 0 & 0 & 0 & 0 & 0 \\
\hline $\mathrm{N} 3$ & 0 & 0 & 0 & 0 & 0 & 0 & 0 & 0 & 0 & 0 \\
\hline $\mathrm{N} 4$ & 0 & 0 & 0 & 0 & 0 & 0 & 0 & 0 & 0 & 0.01 \\
\hline $\mathrm{N} 5$ & 0 & 0 & 0 & 0 & 0.01 & 0.01 & 0.01 & 0.01 & 0.02 & 0.02 \\
\hline $\mathrm{N} 6$ & 0 & 0.01 & 0.02 & 0.03 & 0.03 & 0.04 & 0.05 & 0.05 & 0.05 & 0.06 \\
\hline $\mathrm{N} 7$ & 0.05 & 0.08 & 0.11 & 0.12 & 0.13 & 0.14 & 0.14 & 0.14 & 0.14 & 0.14 \\
\hline $\mathrm{N} 8$ & 0.57 & 0.51 & 0.46 & 0.42 & 0.38 & 0.35 & 0.32 & 0.3 & 0.28 & 0.26 \\
\hline $\mathrm{N} 9$ & 0.57 & 0.51 & 0.46 & 0.42 & 0.38 & 0.35 & 0.32 & 0.3 & 0.28 & 0.26 \\
\hline $\mathrm{N} 10$ & 0.05 & 0.08 & 0.11 & 0.12 & 0.13 & 0.14 & 0.14 & 0.14 & 0.14 & 0.14 \\
\hline $\mathrm{N} 11$ & 0 & 0.01 & 0.02 & 0.03 & 0.03 & 0.04 & 0.05 & 0.05 & 0.05 & 0.06 \\
\hline $\mathrm{N} 12$ & 0 & 0 & 0 & 0 & 0.01 & 0.01 & 0.01 & 0.01 & 0.02 & 0.02 \\
\hline $\mathrm{N} 13$ & 0 & 0 & 0 & 0 & 0 & 0 & 0 & 0 & 0 & 0.01 \\
\hline $\mathrm{N} 14$ & 0 & 0 & 0 & 0 & 0 & 0 & 0 & 0 & 0 & 0 \\
\hline $\mathrm{N} 15$ & 0 & 0 & 0 & 0 & 0 & 0 & 0 & 0 & 0 & 0 \\
\hline $\mathrm{N} 16$ & 0 & 0 & 0 & 0 & 0 & 0 & 0 & 0 & 0 & 0 \\
\hline
\end{tabular}

Table 2 . The data map produced by a unit pulse applied to element E1

\begin{tabular}{|c|l|l|l|l|l|l|l|l|l|l|}
\hline $\mathrm{t}(\mathrm{ms})$ & 0.01 & 0.02 & 0.03 & 0.04 & 0.05 & 0.06 & 0.07 & 0.08 & 0.09 & 0.1 \\
\hline $\mathrm{N} 1$ & 1.14 & 1.02 & 0.92 & 0.83 & 0.76 & 0.70 & 0.64 & 0.60 & 0.55 & 0.52 \\
\hline $\mathrm{N} 2$ & 0.62 & 0.59 & 0.56 & 0.54 & 0.51 & 0.49 & 0.46 & 0.44 & 0.42 & 0.40 \\
\hline $\mathrm{N} 3$ & 0.05 & 0.09 & 0.12 & 0.15 & 0.17 & 0.18 & 0.19 & 0.19 & 0.20 & 0.20 \\
\hline $\mathrm{N} 4$ & 0.00 & 0.01 & 0.02 & 0.03 & 0.04 & 0.05 & 0.06 & 0.07 & 0.07 & 0.08 \\
\hline $\mathrm{N} 5$ & 0.00 & 0.00 & 0.00 & 0.01 & 0.01 & 0.01 & 0.02 & 0.02 & 0.02 & 0.03 \\
\hline $\mathrm{N} 6$ & 0.00 & 0.00 & 0.00 & 0.00 & 0.00 & 0.00 & 0.00 & 0.00 & 0.01 & 0.01 \\
\hline $\mathrm{N} 7$ & 0.00 & 0.00 & 0.00 & 0.00 & 0.00 & 0.00 & 0.00 & 0.00 & 0.00 & 0.00 \\
\hline $\mathrm{N} 8$ & 0.00 & 0.00 & 0.00 & 0.00 & 0.00 & 0.00 & 0.00 & 0.00 & 0.00 & 0.00 \\
\hline $\mathrm{N} 9$ & 0.00 & 0.00 & 0.00 & 0.00 & 0.00 & 0.00 & 0.00 & 0.00 & 0.00 & 0.00 \\
\hline $\mathrm{N} 10$ & 0.00 & 0.00 & 0.00 & 0.00 & 0.00 & 0.00 & 0.00 & 0.00 & 0.00 & 0.00 \\
\hline $\mathrm{N} 11$ & 0.00 & 0.00 & 0.00 & 0.00 & 0.00 & 0.00 & 0.00 & 0.00 & 0.00 & 0.00 \\
\hline $\mathrm{N} 12$ & 0.00 & 0.00 & 0.00 & 0.00 & 0.00 & 0.00 & 0.00 & 0.00 & 0.00 & 0.00 \\
\hline $\mathrm{N} 13$ & 0.00 & 0.00 & 0.00 & 0.00 & 0.00 & 0.00 & 0.00 & 0.00 & 0.00 & 0.00 \\
\hline $\mathrm{N} 14$ & 0.00 & 0.00 & 0.00 & 0.00 & 0.00 & 0.00 & 0.00 & 0.00 & 0.00 & 0.00 \\
\hline $\mathrm{N} 15$ & 0.00 & 0.00 & 0.00 & 0.00 & 0.00 & 0.00 & 0.00 & 0.00 & 0.00 & 0.00 \\
\hline $\mathrm{N} 16$ & 0.00 & 0.00 & 0.00 & 0.00 & 0.00 & 0.00 & 0.00 & 0.00 & 0.00 & 0.00 \\
\hline
\end{tabular}

Table 3. The data map produced by a unit pulse applied to element E2

\begin{tabular}{|c|c|c|c|c|c|c|c|c|c|c|}
\hline $\mathrm{t}(\mathrm{ms})$ & 0.01 & 0.02 & 0.03 & 0.04 & 0.05 & 0.06 & 0.07 & 0.08 & 0.09 & 0.1 \\
\hline $\mathrm{N} 1$ & 0.10 & 0.16 & 0.21 & 0.24 & 0.26 & 0.28 & 0.28 & 0.29 & 0.29 & 0.28 \\
\hline $\mathrm{N} 2$ & 0.57 & 0.52 & 0.48 & 0.44 & 0.41 & 0.39 & 0.37 & 0.35 & 0.33 & 0.32 \\
\hline $\mathrm{N} 3$ & 0.57 & 0.51 & 0.46 & 0.42 & 0.39 & 0.36 & 0.33 & 0.31 & 0.29 & 0.28 \\
\hline $\mathrm{N} 4$ & 0.05 & 0.08 & 0.11 & 0.12 & 0.13 & 0.14 & 0.15 & 0.15 & 0.15 & 0.15 \\
\hline $\mathrm{N} 5$ & 0.00 & 0.01 & 0.02 & 0.03 & 0.03 & 0.04 & 0.05 & 0.05 & 0.06 & 0.06 \\
\hline $\mathrm{N} 6$ & 0.00 & 0.00 & 0.00 & 0.00 & 0.01 & 0.01 & 0.01 & 0.02 & 0.02 & 0.02 \\
\hline $\mathrm{N} 7$ & 0.00 & 0.00 & 0.00 & 0.00 & 0.00 & 0.00 & 0.00 & 0.00 & 0.00 & 0.01 \\
\hline $\mathrm{N} 8$ & 0.00 & 0.00 & 0.00 & 0.00 & 0.00 & 0.00 & 0.00 & 0.00 & 0.00 & 0.00 \\
\hline $\mathrm{N} 9$ & 0.00 & 0.00 & 0.00 & 0.00 & 0.00 & 0.00 & 0.00 & 0.00 & 0.00 & 0.00 \\
\hline $\mathrm{N} 10$ & 0.00 & 0.00 & 0.00 & 0.00 & 0.00 & 0.00 & 0.00 & 0.00 & 0.00 & 0.00 \\
\hline $\mathrm{N} 11$ & 0.00 & 0.00 & 0.00 & 0.00 & 0.00 & 0.00 & 0.00 & 0.00 & 0.00 & 0.00 \\
\hline $\mathrm{N} 12$ & 0.00 & 0.00 & 0.00 & 0.00 & 0.00 & 0.00 & 0.00 & 0.00 & 0.00 & 0.00 \\
\hline $\mathrm{N} 13$ & 0.00 & 0.00 & 0.00 & 0.00 & 0.00 & 0.00 & 0.00 & 0.00 & 0.00 & 0.00 \\
\hline $\mathrm{N} 14$ & 0.00 & 0.00 & 0.00 & 0.00 & 0.00 & 0.00 & 0.00 & 0.00 & 0.00 & 0.00 \\
\hline
\end{tabular}




\begin{tabular}{|l|l|l|l|l|l|l|l|l|l|l|}
\hline N15 & 0.00 & 0.00 & 0.00 & 0.00 & 0.00 & 0.00 & 0.00 & 0.00 & 0.00 & 0.00 \\
\hline N16 & 0.00 & 0.00 & 0.00 & 0.00 & 0.00 & 0.00 & 0.00 & 0.00 & 0.00 & 0.00 \\
\hline
\end{tabular}

Table 4. The data map produced by a unit pulse applied to element E3

\begin{tabular}{|c|c|c|c|c|c|c|c|c|c|c|}
\hline $\mathrm{t}(\mathrm{ms})$ & 0.01 & 0.02 & 0.03 & 0.04 & 0.05 & 0.06 & 0.07 & 0.08 & 0.09 & 0.1 \\
\hline $\mathrm{N} 1$ & 0.01 & 0.02 & 0.04 & 0.05 & 0.07 & 0.08 & 0.09 & 0.10 & 0.11 & 0.12 \\
\hline $\mathrm{N} 2$ & 0.05 & 0.08 & 0.11 & 0.13 & 0.14 & 0.15 & 0.15 & 0.16 & 0.16 & 0.16 \\
\hline $\mathrm{N} 3$ & 0.57 & 0.51 & 0.46 & 0.42 & 0.38 & 0.35 & 0.32 & 0.30 & 0.28 & 0.26 \\
\hline $\mathrm{N} 4$ & 0.57 & 0.51 & 0.46 & 0.42 & 0.38 & 0.35 & 0.32 & 0.30 & 0.28 & 0.26 \\
\hline $\mathrm{N} 5$ & 0.05 & 0.08 & 0.11 & 0.12 & 0.13 & 0.14 & 0.14 & 0.14 & 0.14 & 0.14 \\
\hline $\mathrm{N} 6$ & 0.00 & 0.01 & 0.02 & 0.03 & 0.03 & 0.04 & 0.05 & 0.05 & 0.05 & 0.06 \\
\hline $\mathrm{N} 7$ & 0.00 & 0.00 & 0.00 & 0.00 & 0.01 & 0.01 & 0.01 & 0.01 & 0.02 & 0.02 \\
\hline $\mathrm{N} 8$ & 0.00 & 0.00 & 0.00 & 0.00 & 0.00 & 0.00 & 0.00 & 0.00 & 0.00 & 0.01 \\
\hline $\mathrm{N} 9$ & 0.00 & 0.00 & 0.00 & 0.00 & 0.00 & 0.00 & 0.00 & 0.00 & 0.00 & 0.00 \\
\hline $\mathrm{N} 10$ & 0.00 & 0.00 & 0.00 & 0.00 & 0.00 & 0.00 & 0.00 & 0.00 & 0.00 & 0.00 \\
\hline $\mathrm{N} 11$ & 0.00 & 0.00 & 0.00 & 0.00 & 0.00 & 0.00 & 0.00 & 0.00 & 0.00 & 0.00 \\
\hline $\mathrm{N} 12$ & 0.00 & 0.00 & 0.00 & 0.00 & 0.00 & 0.00 & 0.00 & 0.00 & 0.00 & 0.00 \\
\hline $\mathrm{N} 13$ & 0.00 & 0.00 & 0.00 & 0.00 & 0.00 & 0.00 & 0.00 & 0.00 & 0.00 & 0.00 \\
\hline $\mathrm{N} 14$ & 0.00 & 0.00 & 0.00 & 0.00 & 0.00 & 0.00 & 0.00 & 0.00 & 0.00 & 0.00 \\
\hline $\mathrm{N} 15$ & 0.00 & 0.00 & 0.00 & 0.00 & 0.00 & 0.00 & 0.00 & 0.00 & 0.00 & 0.00 \\
\hline $\mathrm{N} 16$ & 0.00 & 0.00 & 0.00 & 0.00 & 0.00 & 0.00 & 0.00 & 0.00 & 0.00 & 0.00 \\
\hline
\end{tabular}

